\title{
Zinc Oxide Nanorods Prepared in Mixed Solvents
}

\author{
Mohammad Ashraf Shah ${ }^{1,2}$, Fahad M. Al-Marzouki ${ }^{1}$ \\ ${ }^{1}$ Department of Physics, King Abdul Aziz University, Jeddah, Kingdom of Saudi Arabia; ${ }^{2}$ Electron Microscopy Centre, Department \\ of Physics, Faculty of science, National Institute of Technology, Srinagar, India. \\ Email: mashahnit@yahoo.com
}

Received January $9^{\text {th }}, 2010$; revised February $27^{\text {th }}, 2010$; accepted March $2^{\text {nd }}, 2010$.

\begin{abstract}
In this paper, we demonstrate a novel and direct synthesis of hexagonal-shaped zinc oxide ( $\mathrm{ZnO}$ ) nanorods at very low temperature of $\sim 80^{\circ} \mathrm{C}$ simply by using metallic zinc foil and de-ionized $(D I)$ water with few drops of ethanol. The formation of ZnO structures by the reaction of metals with DI water is suggested to occur due to the oxidation of metallic zinc in presence of water. The synthesized $\mathrm{ZnO}$ products were characterized in terms of their structural and optical properties. By the morphological investigations using FESEM, it was observed that the grown products are hexagonal-shaped $\mathrm{ZnO}$ nanorods with the diameters in the range of 50-60 $\mathrm{nm}$ and length with $\sim 1$ micrometer. The EDS and XRD pattern confirmed the composition and crystallinity of the grown nanorods and revealed that the grown products are pure $\mathrm{ZnO}$ with the wurtzite hexagonal phase.
\end{abstract}

Keywords: ZnO, Nanorods, X-Ray Diffraction, Structural Properties

\section{Introduction}

Zinc oxide is rapidly gaining credibility as a material with excellent possibilities for electronic and photonic devices. It exhibits a wide band gap (3.37 eV), large excitation binding energy (60 $\mathrm{meV})$, biocompatibility, and high melting temperature $(2248 \mathrm{~K})$, presenting itself a promising material for wide range of well known technological applications which are well documented [1-4]. Owing to the semiconducting and piezoelectric dual properties of $\mathrm{ZnO}$ crystals, novel applications are introduced which have profound effect in many areas such as self-powdered nanodevices and nanosystems [5]. The demonstration of room temperature ultraviolet lasers, field effect transistors and field emission arrays based on $\mathrm{ZnO}$ nanorods have stimulated great interest in developing functional nanodevices. Moreover, the wide range of morphological diversity in the nano-regime has made this material a promising candidate in the field of nanotechnology and opened up new possibilities for the fabrication of high performance devices based on these nanostructures [6]. Among the various nanoforms, one dimensional (1D) nanostructures have received considerable attention due to their potential interests for understanding fundamental physical concepts and for efficient field emission that has enormous commercial applications such as field emission flat panel displays, x-ray sources, parallel beam electron microscopy and vacuum microwave amplifiers [7].
Apart from commercial methods, recently various new methods have been developed for the synthesis of $\mathrm{ZnO}$ nanostructures. Among the physical methods, chemical and physical vapour deposition, metallorganic vapour phase epitaxy, thermal reduction route, template based method and pyrolysis methods have been used for the successful synthesis of 1D ZnO nanostructures [8-12]. But physical methods generally need expensive equipments, high temperatures and complex producers which restrict further development in actual applications. The chemical methods reported in the literature include decomposition routes of zinc precursor salt, sol-gel and solvothermal process $[13,14]$. These approaches generally make use of frequent amines or other additives that can produce unintentional defects. Therefore, it provides motivation and is desirable for device application point of view to synthesize zinc oxide nanostructures at low temperatures without additives or organics.

In this paper, a versatile and expedient route to grow zinc oxide nanorods by a simple reaction of zinc metal with water at very low temperature of $\sim 80^{\circ} \mathrm{C}$ using few drops of ethanol has been presented. The grown nanorods were characterized in detail in terms of their structural properties. The morphological and structural investigations revealed that the as-grown $\mathrm{ZnO}$ nanorods are hexagonal and possessing well crystallinity with wurtzite hexagonal phase. Finally a plausible growth mechanism has been proposed for the growth of $\mathrm{ZnO}$ nanorods. 


\section{Experimental}

\subsection{Materials}

All the reagents and solvents were of analytical grade and were used without any further purification. Zinc powder was used as a source of Zinc and was cleaned by ultra-sonication in acetone and water for 20 minutes in each solvent.

\subsection{Preparation of the Samples}

In a typical synthesis, appropriate amount of zinc metal foil was taken with $50 \mathrm{ml}$ of distilled water in a Teflon-lined stainless steel chamber with $110 \mathrm{ml}$ capacity. Few drops of ethanol were added to avoid the agglomeration. The prepared reaction mixture was kept at $80^{\circ} \mathrm{C}$ in an oven for 24 hours. After the desired time, the system was allowed to cool down naturally and the resulting mixture was centrifuged. The zinc foils, collected from the reactions vessels, were washed with de-ionized water several times and finally dried in air.

\subsection{Characterization}

The morphology and the size of the products were examined by using field emission scanning electron microscope (FESEM; FEI NOVA NANOSEM-600) coupled with energy dispersive $\mathrm{x}$-ray (EDX) spectrometer. The crystallinity and crystal phases of the grown nanorods were observed by using X-ray diffraction patterns measured with $\mathrm{Cu}-\mathrm{K} \alpha(\lambda=0.15141 \mathrm{~nm})$ radiation (Siemens D 5005 diffractometer).

\section{Results}

\subsection{Morphology of Samples}

The general morphologies of the as-grown structures, obtained after the reaction of zinc foil with water at $80^{\circ} \mathrm{C}$ for $24 \mathrm{~h}$, was observed by FESEM and demonstrated in Figure 1 which confirms that the grown products are hexagonal nanorod shaped. Figure 1(a) and (b) show the low and high magnification FESEM images of the nanorods and confirms that the nanorods are grown in a very high density over the whole foil substrate. The typical lengths of the grown nanorods are $1 \mu \mathrm{m}$. The typical diameters of the as-grown nanorods are $\sim 60 \pm 10 \mathrm{~nm}$. The nanorods are exhibiting hexagonal surfaces and facets throughout their lengths which confirm that the nanorods are well-crystalline.

\subsection{X-Ray Dispersive Analysis}

To check the composition of the as-grown nanorods, EDX analysis was performed. Figure 2 demonstrates the typical $\mathrm{EDX}$ analysis of the as-grown $\mathrm{ZnO}$ nanorods. It is confirmed from the EDX analysis that the grown nanorods are composed of zinc and oxygen only. The molecular ratio of $\mathrm{Zn}: \mathrm{O}$ of the grown nanorods, calculated from EDX and quantitative analysis data, is close to that of 1:1. Except $\mathrm{Zn}$ and $\mathrm{O}$, no other peak for any other element has been found in the spectrum which again confirmed that the grown nanorods are pure $\mathrm{ZnO}$.

\subsection{X-Ray Diffraction Analysis}

To identify the crystallinity and crystal phases of the as-grown structures, $\mathrm{X}$-ray diffraction (XRD) analysis was performed and shown in Figure 1. Figure 1 shows the typical XRD pattern of the as-grown nanostructures on zinc foil. All the peaks in the pattern can be indexed to hexagonal wurtzite structure with space group $\mathrm{P} 6{ }_{3} \mathrm{mc}$ and lattice constants $a=0.3249 \mathrm{~nm}, c=0.5206 \mathrm{~nm}$, (JCPDS card no. 36-1451). No diffraction peaks arising from any impurity can be detected in the pattern confirms that the grown products are pure $\mathrm{ZnO}$.

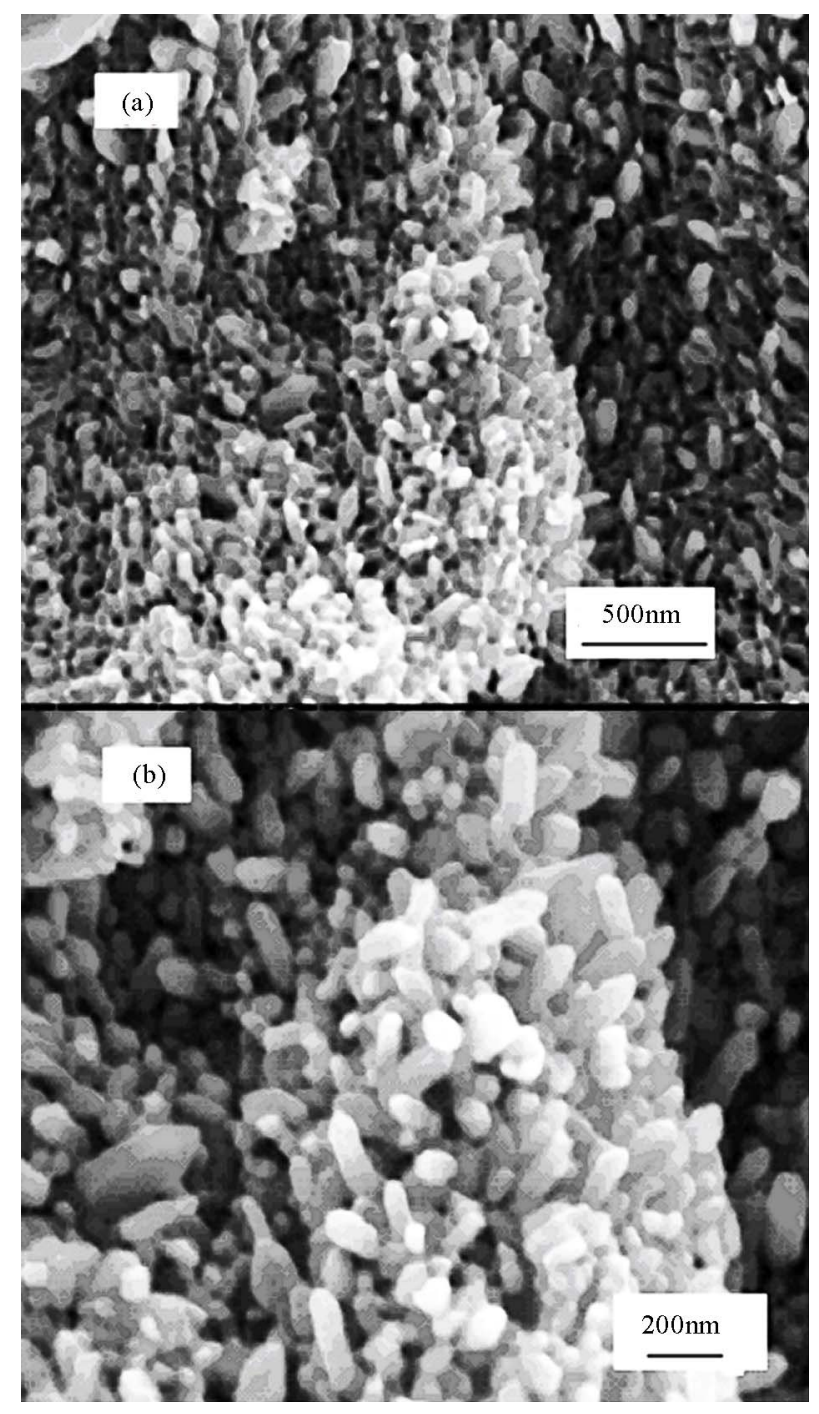

Figure 1. Typical (a) low and (b) high-resolution FESEM images of nanorods obtained by the reaction of zinc metal powder with water at $80^{\circ} \mathrm{C}$ for $24 \mathrm{~h}$ 
Label A:

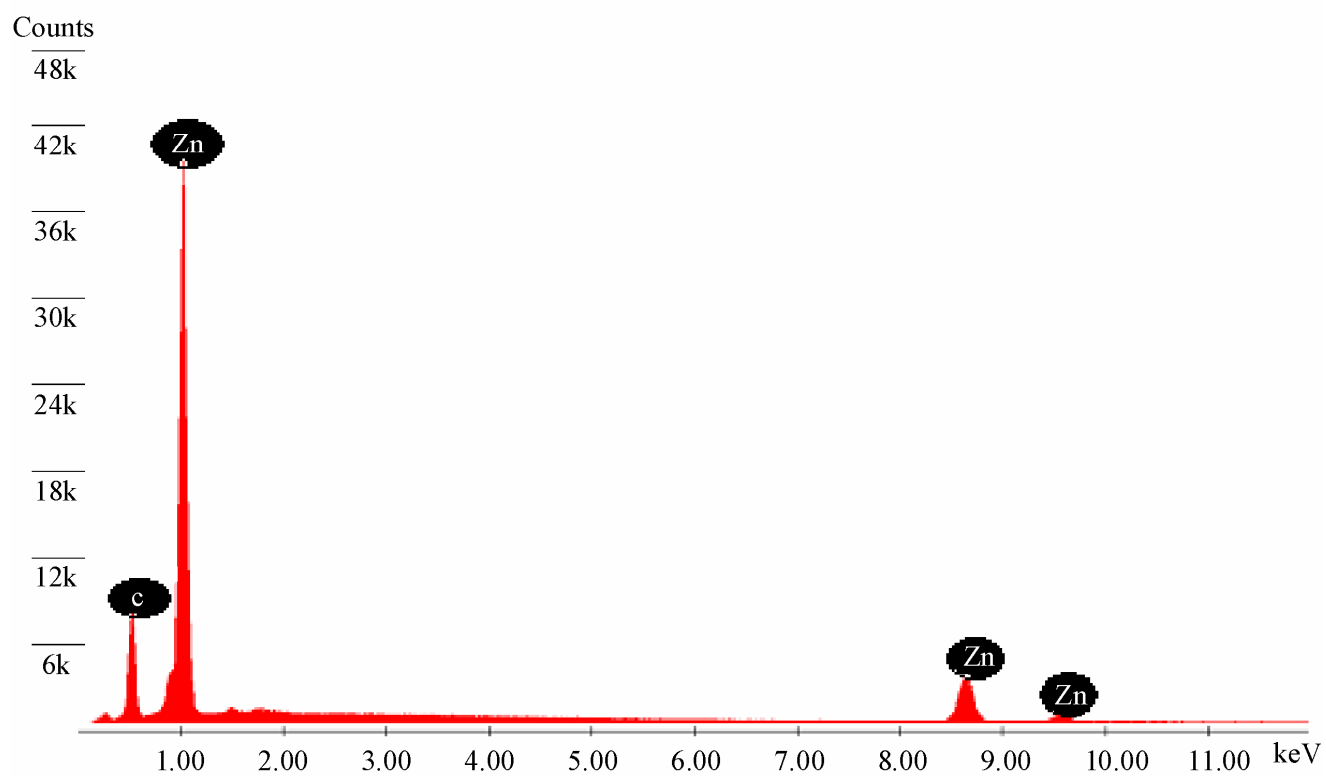

Figure 2. The corresponding EDX analysis confirming the existence of all elements involved in sample preparation

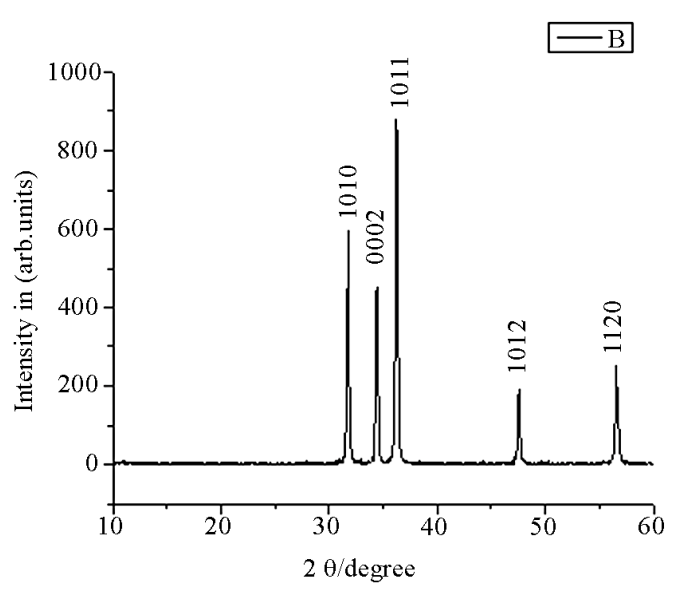

Figure 3. XRD pattern of zinc oxide nanorods

\section{Discussion}

The formation of $\mathrm{ZnO}$ nanorods on zinc foil in the presence of water can be explained by various chemical reactions. As initially, zinc does not react with water molecules but at $80^{\circ} \mathrm{C}$ and under pressure in Teflon-lined stainless chamber, the zinc reacted with water and forms a protective zinc hydroxide $\left(\mathrm{Zn}(\mathrm{OH})_{2}\right)$ layer with dissolved hydroxide ions onto the surfaces of the zinc foil according to the following reaction mechanism.

$$
\mathrm{Zn}^{2+}+\mathbf{2 0 H ^ { - }} \rightarrow \mathbf{Z n}(\mathrm{OH})_{2} \text { (s) }
$$

Moreover, as the concentration of the $\mathrm{Zn}^{2+}$ and $\mathrm{OH}^{-}$ions exceeds a critical value, the precipitation of $\mathrm{ZnO}$ nuclei starts. The $\mathrm{Zn}(\mathrm{OH})_{2}$ can be transformed into the $\mathrm{ZnO}$ crystals via the simple chemical reactions mentioned below:

$$
\mathrm{Zn}(\mathrm{OH})_{2} \stackrel{\triangle}{\longrightarrow} \mathrm{ZnO}+\mathrm{H}_{2} \mathrm{O}
$$

The precipitates of $\mathrm{Zn}(\mathrm{OH})_{2}$ are more soluble as compared to the $\mathrm{ZnO}$ precipitates, therefore, the formed $\mathrm{Zn}(\mathrm{OH})_{2}$ precipitates tend to continuously produce $\mathrm{Zn}^{2+}$ and $\mathrm{OH}^{-}$ions which form the $\mathrm{ZnO}$ nuclei. The formed $\mathrm{ZnO}$ nuclei are the building blocks for the formation of the final products. With increasing the reaction time, the deposition over the $\mathrm{ZnO}$ nuclei increases in uni-direction and finally $\mathrm{ZnO}$ nanorods were deposited over the $\mathrm{Zn}$-foil substrates. Even though a plausible growth process for the formation of $\mathrm{ZnO}$ hexagonal-shaped $\mathrm{ZnO}$ nanorods are described here but more studies are needed to clearly explain the growth process for the formation of these nanorods.

\section{Conclusions}

An efficient and expedient route has been explored for the synthesis of $\mathrm{ZnO}$ nanorods at low temperature without additives and surfactants. The proposed single source and catalyst free method is simple, economic and environmentally benign which will make it suitable for various $\mathrm{ZnO}$ nanorods based applications.

\section{Acknowledgements}

We are also pleased to acknowledge World Bank for their financial support in procuring Scanning Electron Microscopy. 


\section{REFERENCES}

[1] C. X. Xu, X. W. Sun, Z. L. Dong, M. B. Yu, T. D. My, X. H. Zhang, S. J. Chua and T. T. White, "Zinc Oxide Nanorods and Nanowires Fabricated by Vapor Phase Transport at Low Temperature," Nanotechnology, Vol. 15, No. 7, 2004, pp. 839-842.

[2] C. Xu, G. Xu, Y. Liu and G. Wang, "A simple and novel route for the preparation of $\mathrm{ZnO}$ nanorods," Solid State Communication, Vol. 122, No. 2-4, 2002, pp. 175-179.

[3] H. Wie, Y. Wu, N. Lun and C. Hu, "Hydrothermal Synthesis and Characterization of Zno Nanorods," Materials Science and Engineering A, Vol. 393, No. 1-2, 2005, pp. 80-82.

[4] Z. W. Pan, Z. R. Dia and Z. L. Wang, "Nanobelts of Semiconducting Oxides," Science, Vol. 291, No. 5510, 2001, pp. 1947-1949.

[5] X. Wang, J. Sang and Z. L. Wang, "Nanowires and Nanobelt Arrays of Zinc Oxide from Synthesis to Properties and to Novel Devices," Journal of Materials Chemistry, Vol. 17, 2007, No. 8, pp. 711-720.

[6] Y. H. Yang, D. Wang and G. W. Wang, "Growth Mechanism of One-Dimensional Hierarchical Structures," Nanotechnology, Vol. 17, No. 2, 2006, pp. 5556-5560.

[7] W. I. Park, D. H. Kim, S. W. Jung and G. C. Yi, "Metallo-Organic Vapor Phase Epitaxial Growth of Vertically Aligned Zno Nanorods," Applied Physic Letters, Vol. 80, No. 22, 2002, pp. 4232-4234.
[8] Y. W. Koh and K. P. Loh, "Hexagonally Packed Zinc Oxide Nanorods Bundles on Hydrotalcide Sheets," Journal of Materials Chemistry, Vol. 15, 2005, p. 2508.

[9] R. Muller, L.Madler and S. E. Pratginis, "Nanoparticle Synthesis at High Production Rate by Flame Spray Pyrolysis," Chemical Engineering Science, Vol. 58, No. 10, 2003, pp. 1969-1976.

[10] Y. Yang and H. Chen, "Size Control of Zinc Oxide Nanoparticles Via Thermal Decomposition of Zinc Acetate Coated on Organic Additives," Journal of Crystal Growth, Vol. 263, No. 1-4, 2004, pp. 447-453.

[11] M. A. Shah and M. A. Asiri, "Simple route for Zinc oxide nanorods," International Journal of Nanoparticles, Vol. 2 No. 2, 2009, p. 49.

[12] M. N. Kamalasanan and S. Chandra, "Sol-Gel Synthesis of Zno Thin Films," Thin solid Film, Vol. 288, 1996, pp.112-115.

[13] Q. Ahsanulhaq, A Umar and Y. B Hahn, "Growth of Aligned Zno Nanorods And Nanopencils On Zno/Si In Aqueous Solution, Growth Mechanism and Structural And Optical Properties," Nanotechnology, Vol. 18, No. 11, 2007, p. 115603.

[14] D. Ying, Y, Zhang, Y. Q. Bai, Z. L. Wang, "Bicrystalline Zinc Oxide Nanowires," Chemical Physics Letters, Vol. 375, No. 1-2, 2003, pp. 96-101. 